Research Article

\title{
Application of Improved Combined Deterministic-Stochastic Subspace Algorithm in Bridge Modal Parameter Identification
}

\author{
Peng Wen, ${ }^{1}$ Inamullah Khan $\mathbb{D D}^{2},{ }^{2}$ Jie He, ${ }^{3}$ and Qiaofeng Chen ${ }^{1}$ \\ ${ }^{1}$ Bridge Engineering Department, Southwest Jiaotong University, Chengdu, China \\ ${ }^{2}$ School of Civil Engineering, National University of Sciences and Technology, Islamabad, Pakistan \\ ${ }^{3}$ China Railway Eryuan Engineering Group. Co. Ltd, Chengdu, China \\ Correspondence should be addressed to Inamullah Khan; inam_bunny@yahoo.com
}

Received 18 August 2020; Revised 16 February 2021; Accepted 3 March 2021; Published 11 March 2021

Academic Editor: Pedro A. Costa

Copyright (C) 2021 Peng Wen et al. This is an open access article distributed under the Creative Commons Attribution License, which permits unrestricted use, distribution, and reproduction in any medium, provided the original work is properly cited.

Modal parameter identification is considered to be one of the most important tasks in structural health monitoring because it provides a reliable reference for structural vibration control, damage severity, and operational state. Moreover, at present, the combined deterministic-stochastic subspace algorithm is cogitated as one of the key algorithms in the modal parameter identification, which is why it is widely used in the modal parameter identification of bridge structures. In this paper, a novel method is proposed, which is a time-domain identification algorithm, based on sliding window-fuzzy C-means clustering algorithm-combined with deterministic-stochastic subspace identification (SC-CDSI), to achieve online intelligent tracking and identification of modal parameters for nonlinear time-varying structures. First of all, to realize the online tracking and identification process, it is necessary to divide the input and output signal of the nonlinear time-varying structure by windowing; for that, to determine the window function, window size and window step length according to the characteristics of the signal are analyzed. Secondly, in order to satisfy the intelligent identification of effective modals in stability diagram, the fuzzy C-means clustering algorithm is kept as a base, whereas frequency, damping ratio, and modal shapes serve as clustering elements, applied to fuzzy C-means clustering algorithm, and then the intelligent selection of effective modals is achieved. Finally, a shaking table test bridge is used as a modal parameter identification in lab, and its results are compared with the MIDAS finite element results. The compared results show that the proposed SC-CDSI identification algorithm can accurately achieve the intelligent identification of online tracking of the structural frequency, and the identification results are reliable to be used in real-life bridge structures.

\section{Introduction}

Stochastic subspace identification (SSI) [1] is one of the most widely used modal parameter identification algorithms, which can effectively identify the natural frequencies, damping ratios, and modal shapes of bridge structures under environmental excitation [2]. Along with the popularization and development of SSI algorithm, it has also got some limitations. According to a previous study [3], a corresponding improved algorithm for these limitations in SSI was proposed. However, the input signal of recognition system is environmental excitation (the signal is unknown); thus, a clear incentive-system-response model cannot be established in the decomposition process of the modal parameters and can lead to an expansion of the system's eigenvalue solution space. Thus, false modals can exist in the final recognition results, and thereby the accuracy of the recognition results will eventually be reduced.

The combined deterministic-stochastic subspace identification (CDSI) method [4] is established on the basis of SSI algorithm theory. CDSI uses the structure's input signals (excitation) and output signals (response) as the input of the system for parameters identification. They can provide effective remedy for the limitations present in SSI algorithm, which in turn can improve the accuracy of the process. Additionally, the advantages of the CDSI algorithm are that it can fully account the inputs, outputs, and noise of the structure, which is why it is popular among the users. Belleri et al. [5] applied CDSI to concrete frame structures to 
perform an accurate identification of modal parameters in different damage states under earthquake excitation; Bakir [6] applied CDSI to identify frame structures under earthquake excitation. These recognition results show that this approach can effectively identify the natural frequency of the structure, and the identified modal shapes are reliable. Huang et al. [7] applied CDSI to multistory steel frame shaking table test to identify the different states of modal parameters. Thus, it can be concluded that CDSI can be used to the determine the structural damage state.

In addition, Damore et al. [8] adopted the CDSI method to accurately identify the natural vibration frequency of the shaking table test model, which is a 6-layer steel frame.

Thai et al. [4] conducted an experiment on the Walnut Creek Bridge and revealed that the combined deterministicstochastic subspace method could accurately obtain the frequency information of the structure and the identification result was more ideal than that of the stochastic subspace identification.

Moaveni and Asgarieh [9] applied the identification method of definite combined deterministic-stochastic subspace to identify the time-varying nonlinear structural system and compared its identification results with the results of wavelet transformation and finite element analysis.

Lopes Dos Santos [10] introduced a recursive subspace identification algorithm, which is similar to the bilinear system identification algorithm based on Picard iterative process, and this iterative method effectively overcomes the shortcomings of LPV subspace algorithms.

Reynders and Roeck [11] improved the deterministicstochastic subspace algorithm and applied the new algorithm to identify the modal parameters of the Z24 bridge on the A1 highway between Bern and Zurich. As compared to the traditional algorithm, the new algorithm has the advantage of faster operation rate and gives more accurate identification results.

SSI algorithm is widely used in modal parameters identification of bridge structures, but CDSI algorithm is rarely used in modal parameter identification of bridge structures. The main reason is that the core algorithm of CDSI is a linear algorithm [12], which is not suitable for nonlinear time-varying structures like bridge structures. Based on this approach, the sliding window technique [13] is nested within CDSI algorithm to perform the time-varying modal parameter tracking and identification. Additionally, to avoid manual involvement of distinguishing true and false modes, a fuzzy C-means (FCM) algorithm [14] is adopted for intelligent screening of modal parameter results to complete online tracking and intelligent identification of modal parameters.

\section{Basic Principle of CDSI Algorithm}

The flowchart of the CDSI algorithm can be seen in Figure 1, where $u_{k}$ is the input signal of the system; $B$ is input matrix; $w_{k}$ represents various types of noise derived from surrounding circumstances; $x_{k}$ is state vector of the system; $A$ is state matrix; $C$ is output matrix; $v_{k}$ is measured noise derived

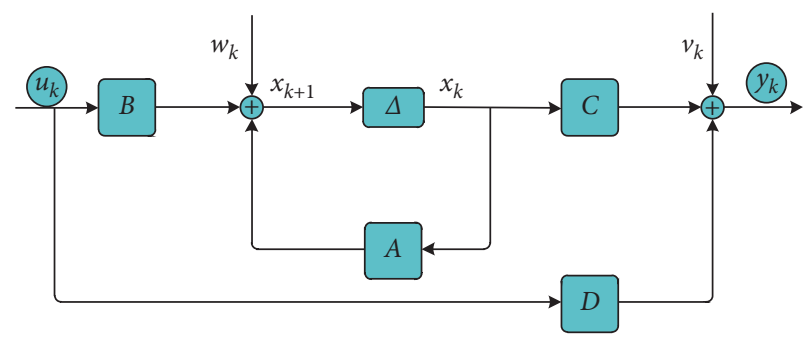

FIgURE 1: Flowchart of the combined deterministic-stochastic system.

from sensors, $D$ is midway transfer matrix, and $y_{k}$ is output data of the system.

A combined deterministic-stochastic state equation is obtained and can be written as

$$
\left(\begin{array}{l}
x_{k+1}=A x+B u_{k}+w_{k} \\
y_{k}=C x_{k}+\mid D u_{k}+v_{k}
\end{array}\right.
$$

Now, to determine the natural frequency, damping ratio, and modal shape, a stepwise equation will be presented in detail:

Step 1: according to the input and output signals, the Hankel matrix is established, where $U_{P}$ is past input data and $U_{f}$ is future output data.

$$
U_{0 \| 2 i-1}=\left|\begin{array}{cccc}
u_{0} & u_{1} & \cdots & u_{j-1} \\
u_{1} & u_{2} & \cdots & u_{j} \\
\cdots & \cdots & \cdots & \cdots \\
u_{i-1} & u_{i} & \cdots & u_{i+j-2} \\
\hline u_{i} & u_{i+1} & \cdots & u_{i+j-1} \\
u_{i+1} & u_{i+2} & \cdots & u_{i+j} \\
\cdots & \cdots & \cdots & \cdots \\
u_{2 i-1} & u_{2 i} & \cdots & u_{2 i+j-2}
\end{array}\right|=\left(\frac{U_{p}}{U_{f}}\right) .
$$

Step 2: to determine the state sequence, it can be written as

$$
X_{i}=\left(\begin{array}{lllll}
x_{i} & x_{i+1} & \cdots & x_{i+j-2} & x_{i+j-1}
\end{array}\right) .
$$

Step 3: now to set up a linear equation containing a state matrix $(A)$, an input matrix $(B)$, an output matrix $(C)$, and a midway transfer matrix $(D)$, it can be written as

$$
\left[\begin{array}{c}
\widetilde{X}_{i+1} \\
Y_{i+1}
\end{array}\right]=\left[\begin{array}{cc}
A & B \\
C & D
\end{array}\right]\left[\begin{array}{c}
\widetilde{X}_{i} \\
U_{i / i}
\end{array}\right]+\left[\begin{array}{c}
\rho_{w} \\
\rho_{v}
\end{array}\right] .
$$

In the above formula, $\rho_{w}$ and $\rho_{w}$ are residuals, where both are orthogonal to $\bar{X}_{i}$ and $\bar{X}_{i+1}$. 
Step 4: now eigenvalue decomposition [15] of matrix $A$ is performed and can be written as

$$
\begin{aligned}
A & =e^{A_{c} \Delta t}, \\
\lambda_{i} & =e^{\lambda_{i}^{c} \Delta t}, \\
\lambda_{i}^{c} & =\frac{\operatorname{In} \lambda_{i}}{\Delta t}, \\
\lambda_{i}^{c}, \bar{\lambda}_{i}^{c} & =-\xi_{i} \omega_{i} \pm j \omega_{i} \sqrt{1-\xi_{i}^{2}} .
\end{aligned}
$$

Step 5: according to formula (6), the natural frequency value $\left(\left(f_{j}-f_{j+1}\right) / f_{j}\right) \times 100 \%<\Delta_{f}(\diamond \diamond)$, damping ratio (MAC), and modal shape $(\Phi)$ of the structure are performed.

$$
\begin{aligned}
& w_{i}=\sqrt{a_{i}^{2}+b_{i}^{2}}\left(\frac{\mathrm{rad}}{\mathrm{s}}\right), \\
& f_{i}=\frac{\sqrt{a_{i}^{2}+b_{i}^{2}}}{2 \pi}(\mathrm{Hz}), \\
& \Phi=C \Psi .
\end{aligned}
$$

In the above formula, $\psi$ is the eigenvector matrix of the system, and it is also a complex number.

\section{Application of Sliding Window Technique}

3.1. Addition of Windows Analysis. In the process of modal parameter identification of the response signal, the efficiency of parameter identification can be improved by controlling the length of the signal; therefore, in practical application, the length of the signal can be controlled by windowing truncation processing. Additionally, the length of signal can be controlled by windowing and truncating process. However, the window functions will directly correspond to the signal's spectrum in the process on windowing. Different window forms cause different levels of spectrum leakage, and that is why the final frequency resolution effect will be different. Based on these conditions, it is particularly important to choose the type of window functions, the size of the windows, and the step length of sliding window.

3.2. Window Function Selection. Currently, commonly used window functions [16] are rectangular window, Hanning window, Hamming window, flat-topped window, Gaussian window, and so forth. Comparing the advantages and disadvantages of each window function, a rectangular window was selected as the windowing function of the response signal in this paper. Since the rectangular window suits better for our analysis due to its simplicity and accuracy in frequency identification, it is used in this paper. In addition, rectangular window is a zero-power window of time variable; it also has the advantage of main lobe concentration and can also accurately identify the frequency results, but its accuracy of amplitude identification is a little bit low, which does not have much impact on the analysis of this paper. Therefore, if it is to be only used for accurate identification of frequency main lobe and does not require the accuracy of amplitude recognition, which is the case in a practical application, such as identifying the natural frequency of the structure, a rectangular function can be preferably selected for windowing of signal. The rectangular window function can be written as

$$
w(t)=1
$$

3.3. Window Size Determination. The window size and the quantity of initial data modus operand once entered into system each time will definitely affect the accuracy of the recognition results up to certain extent. If the window size is too small, the accurate stability diagram cannot be identified due to insufficient input signals and thus will easily result in mode omissions. However, if window size is too large, there will be too many false modes into the stability diagram because of too many input signals and will thereby reduce the efficiency of parameters identification process. Based on this scenario, some scholars [17] believe that the characteristics of the structure itself, the sampling frequency of the signal, and the noise level of the signal should be comprehensively considered. However, according to a previous study, it is not feasible to comprehensively determine the size of the window based on these factors, because there are too many factors involved, and each structure has its own unique characteristics. Therefore, the size of window can be defined by referring to the principle of order determination of stability diagram. For that, specific analysis steps are required, which are as follows:

(1) The true order Nof the structure is determined based on the order determination [18] of the stability diagram in the stochastic subspace method.

(2) Assume that the initial window data length is $L_{1}$, corresponding window time is $t_{1}$, and sampling frequency is $c f$; the relationship among these three is $L_{1}=t_{1} \times c f$.

(3) It is assumed that the lag time between previous and latter windows is $T$; that is, the data length corresponding to the $i$ th window is $L_{i}=\left[t_{1}+(i-1) \times\right.$ $T] \times c f$.

(4) The corresponding stability diagrams of each window are identified by CDSI algorithm; in case the system order is $\mathrm{N}$, the numbers of corresponding stable points $F_{i}=f_{i}^{1}, f_{i}^{2}, f_{i}^{3}$ to the first 3 modes of each stability diagram are to be calculated, because the order of the system is $N$. The maximum number of stable points corresponding to each mode is $N / 2$; thus the percentages $\mathrm{BF}_{i}=\left(2 f_{i}^{1} / N\right),\left(2 f_{i}^{2} / N\right),\left(2 f_{i}^{3} /\right.$ $N$ ) of the first three modes of stable points can be calculated.

(5) The $\mathrm{BF}_{i}$ numerical value changes with the signal length; due to this limitation, the percentage shows a gradual increase at first and then keeps a steady 
change, as shown in Figure 2. It is considered that once the percentage exceeds $90 \%$, the corresponding signal length is considered to be a suitable window size.

3.4. Window Step Size. Currently, the window size is determined, and still further work is required to determine the sliding step size of the window, that is, the data points moving forward by each window to achieve real-time update of the input signal. Assuming that the quantity of updated data of signal is $V$, the relationship between the data replacement of each window and the input and output of the system are shown in Figure 3.

According to the definition of the Hankel matrix [19], the length of past input signal is equal to the length of future output signal. Based on this situation, $V$ will be defined as the data quantity corresponding to $1 / 2$ window; that is, the overlap between windows is $1 / 2$ window.

\section{Application of FCM Clustering Algorithm}

SSI algorithm and CDSI algorithm involve manual participation to select effective modals [20] in stability diagram. To avoid subjectivity caused by manual participation in effective modal parameter identification, an intelligent screening method for effectiveness modal selection based on FCM clustering algorithm is proposed. The basic principle and steps of FCM clustering algorithm and then the intelligent identification of effective modes will be determined by combining the FCM algorithm with CDSI algorithm.

4.1. The Basic Principle of FCM Clustering Algorithm. Compared with K-means clustering and pedigree clustering algorithm, FCM clustering [21] has many advantages.

The clustering principle of FCM is based on the degree of membership of the data points to identify its classification; therefore, it is a type of a fuzzy clustering algorithm [22] and can perform clustering of different dimensions of data. To achieve this, the data set $X$ is divided into class $c$ using FCM algorithm, assuming that $X$ consists of $N$ groups sample; that is, $X=\left\{x_{1}, x_{2}, \ldots, x_{N}\right\}$, where each sample consists of $n$-dimensional vectors; that is, $x_{j}=\left\{x_{j 1}, x_{j 2}, \ldots, x_{j n}\right\}$. The membership matrix $U=\left[u_{i j}\right]$ of the data sets $X$ is defined as a matrix of $C \times N$, where $C$ is the number of classifications, and $u_{i j}$ is the $j$ th sample in $X$; the membership belongs to grade $i^{\text {th }}$, and it must meet the following conditions:

$$
\sum_{i=1}^{c} u_{i j}=1, \quad \forall j=1,2, \ldots, N, 0 \leq u_{i j} \leq 1,1 \leq i \leq c, 1 \leq j \leq N
$$

whereas the formulation of $u_{i j}$ is performed as follows:

$$
u_{i j}=\frac{1}{\sum_{k=1}^{c}\left(d_{i j} / d_{k i}\right)^{2 /(m-1)}}, \quad i=1,2, \ldots, c ; j=1,2, \ldots, n .
$$

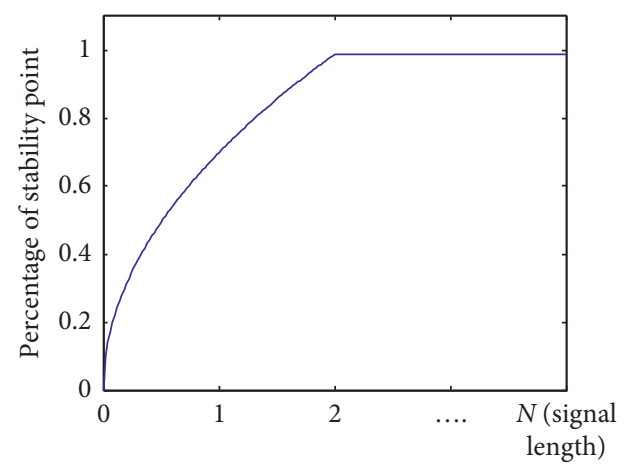

FIgURe 2: Percentage trend chart of stability points.

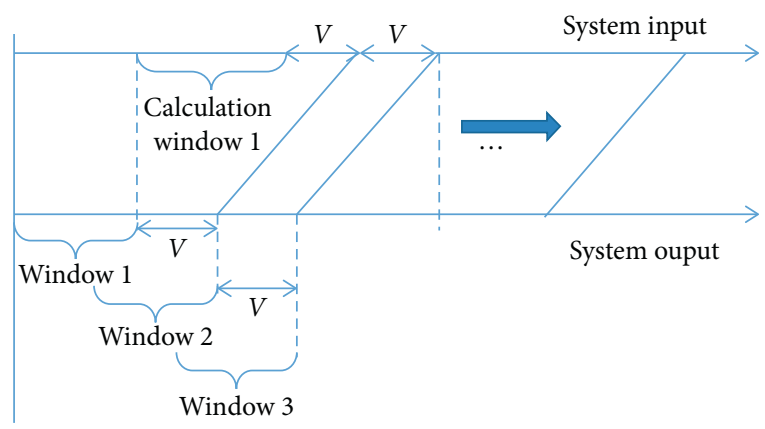

FIgURE 3: Schematic diagram of step length.

Fuzzy index $m \in[1, \infty]$ and $d_{i j} \in\left\|x_{j}-v_{i}\right\|$ are represented in the Euclidean distance between the data object $x_{j}$ and clustering center $v_{i}$ of $i$ th cluster, where $\left\{v_{1}, v_{2}, \ldots, v_{i}\right\}$ represents the clustering center set and its formulation is performed as follows:

$$
v_{i}=\frac{\sum_{j=1}^{n} u_{i j}^{m} \times x_{j}}{\sum_{j=1}^{n} u_{i j}^{m}} .
$$

The objective function value of FCM algorithm is $J(u, v)$; its purpose of iterative process is to find the membership matrix and clustering center to minimize the function $J(u, v)$.

$$
J(u, v)=\sum_{j=1}^{n} \sum_{i=1}^{c} u_{i j}^{m} d^{2}\left(x_{j}, v_{i}\right)
$$
follows:

The specific steps of FCM clustering algorithm are as

(1) The amount of classification $c$ and the maximum number of iterations $m$ (fuzzy index) are determined,

(2) Randomly select a data set from the data sets $X$ as the center point of the initial clustering,

(3) The membership matrix $U$ is performed according to formula (9),

(4) The clustering center $v$ is performed according to formula (10),

(5) According to formula (11), membership matrix and clustering center are calculated, so that the function 
$J(u, v)$ has the minimum value and thus the clustering process got completed.

4.2. Combination of FCM Algorithm and CDSI Algorithm. To effectually realize the intelligent identification of modal in the stability diagram, a new parameter identification algorithm (C-CDSI) is proposed by combining FCM algorithm and CDSI algorithm. The specific steps for achieving such algorithm are as follows.

4.2.1. Identification of Parameters of Each Window. The parameter results $X_{i}=\left\{F_{i}, w_{i}, \Phi_{i}\right\}$ corresponding to each window are identified by CDSI algorithm, where $F$ is frequency value, $w$ is damping ratio, and $\Phi$ is modal shape. Each parameter result is a matrix with $m \times n$, where the value $m$ is equal to $1 / 2$ of the real order.

$$
\begin{aligned}
F_{i} & =f_{i}(m, n), \\
w_{i} & =\xi_{i}(m, n), \\
\Phi_{i} & =\psi_{i}(m, n) .
\end{aligned}
$$

4.2.2. Frequency Clustering. Accordingly, the frequency, damping ratio, and modal shape of $X_{i}=\left\{F_{i}, w_{i}, \Phi_{i}\right\}$ and $X_{j}=\left\{F_{j}, w_{j}, \Phi_{j}\right\}$ are clustered by FCM algorithm. In order to achieve this, the following steps will be followed:

(1) The clustering data set is determined, and it is assumed that the modal frequencies of $f_{1}(m, n)$ and $f_{2}(m, n)$ are clustered.

(2) The number of clustering classifications is determined; because there are two types of modal frequency of the data set for each clustering, the number of classifications is set as $c=2$.

(3) The maximum iterations are determined; because the rows in parameter results matrix are true order $N$ of $1 / 2$ of the system, the maximum iteration is set as $m=N / 2$.

(4) The first-order frequency results $f_{1}(m, 1)$ in $f_{1}$ and the first-order frequency results $f_{2}(m, 1)$ in $f_{2}$ are clustered. If these are in the same category, the average value will be the new first-order frequency result; that is, $f_{1-2}(m, 1)=\left(f_{1}(m, 1)+f_{2}(m, 1)\right) / 2$. All of these modes in the frequency matrix will be clustered and so on. Then, a new frequency matrix $f_{1-2}$ will be established by the clustered new modes with that of the nonclustered modes.

4.2.3. Damping Ratio Clustering. The damping ratio clustering identification of corresponding two-order modes in $\xi_{1}(m, n)$ and $\xi_{2}(m, n)$ is performed. To verify the authenticity of the clustered two-order modes in frequency matrix $f_{1-2}$, the following steps will be followed:

(1) Assuming that $f_{1}(m, 1)$ and $f_{2}(m, 1)$ in $f_{1-2}$ are similar items, the clustering is completed based on Section 4.2.2.
(2) If these are similar terms, the average value will be a new first-order damping ratio result; $\xi_{1-2}(m, 1)=$ $\left(\xi_{1}(m, 1)+\xi_{2}(m, 1)\right) / 2$.

(3) If these are not similar terms, $f_{1-2}(m, 1)$ will be split into the initial items $f_{1}(m, 1)$ and $f_{2}(m, 1)$, and then a new frequency matrix $q_{1-2}$ will be reconstructed.

(4) A new damping ratio matrix $\xi_{1-2}$ is formed from all the clustered damping ratios and the nonclustering damping ratios.

4.2.4. Modal Mode Clustering. The modal shape clustering of the corresponding two-order modes in $\psi_{1}(m, n)$ and $\psi_{2}(m, n)$ is performed; to verify the authenticity of the clustering results of frequency cluster in $q_{1-2}$ and damping ratio cluster in $\psi_{2}(m, n)$, the following steps will be followed:

(1) Assuming that $f_{1}(m, 1)$ and $f_{2}(m, 1)$ in $q_{1-2}$ are similar items, the clustering identification of $\psi_{1}(m, 1)$ and $\psi_{2}(m, 1)$ is completed based on Section 4.2.2.

(2) If these are similar terms, the average value will be a new first-order modal shape result; $\psi_{1-2}(m, 1)=\left(\psi_{1}\right.$ $\left.(m, 1)+\psi_{2}(m, 1)\right) / 2$.

(3) If these are not similar terms, $f_{1-2}(m, 1)$ will be split into the initial items $f_{1}(m, 1)$ and $f_{2}(m, 1)$, and then a new frequency matrix $Q_{1-2}$ will be reconstructed. Additionally, $\xi_{1-2}(m, 1)$ will be split into the initial items $\xi_{1}(m, 1)$ and $\xi_{2}(m, 1)$, and then a new damping ratio matrix $\zeta_{1-2}$ will be reconstructed.

(4) A new modal shape matrix $\psi_{1-2}$ is formed from all the clustered modal shapes and the nonclustering modal shapes.

4.2.5. Clustering of Each Window Parameter Successively. A new set of parameter results $X_{1-2}=\left\{Q_{1-2}, \xi_{1-2}, \psi_{1-2}\right\}$ are established by $Q_{1-2}, \zeta_{1-2}$, and $\psi_{1-2}$, and the same modal cluster between $X_{1-2}$ and $X_{3}$ based on Section 4.2.2 to Section 4.2.4 is completed to obtain a new clustering result $X_{2-3}$. Based on this approach, the parameter result clustering among all windows is hereby termed as completed. Assuming that the total number of windows is $N$, the final clustering parameter result will be $X_{N-1-N}$.

The effective modal parameters identification (the frequency, damping ratio, and modal shape are stable modals) in $X_{N-1-N}$ can be performed by counting each cluster modal of $X_{N-1-N}$, which is made up of the corresponding modals of windows. If modal's clustering window is greater than $0.8 \mathrm{~N}$, it will be an effectiveness modal and will be drawn in stability diagram.

Based on the above descriptions, a new modal parameters online intelligent identification algorithm (SC-CDSI) that combines sliding window technology, FCM clustering algorithm, and CDSI algorithm is proposed. The specific steps of this method are shown in the flowchart diagram in Figure 4. 


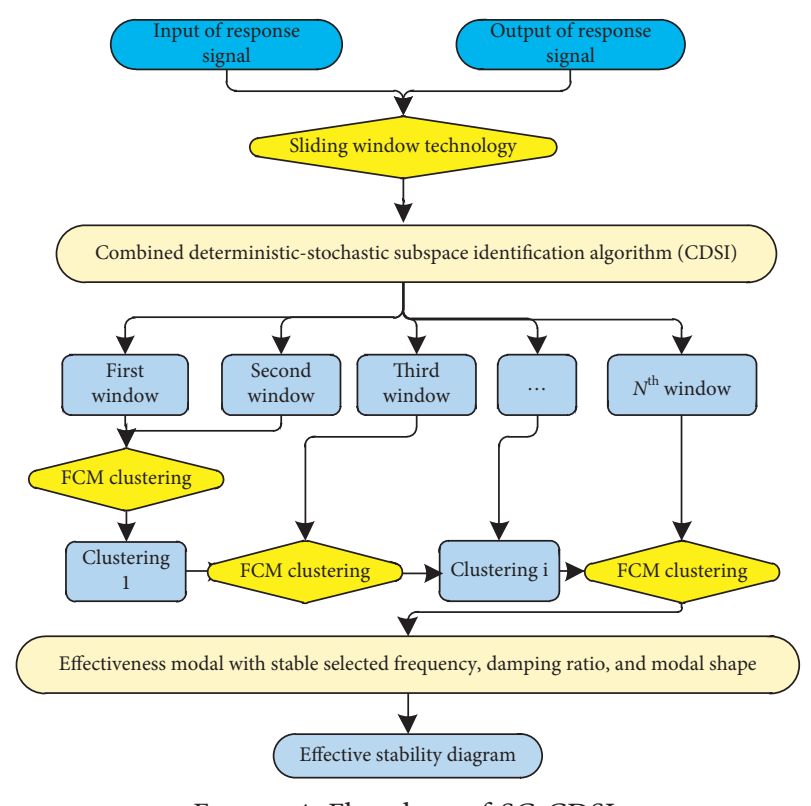

FIgURE 4: Flowchart of SC-CDSI.

\section{Shaking Table Test Bridge}

In order to verify the reliability of the improved combined deterministic-stochastic subspace algorithm (SC-CDSI) as mentioned above, a shaking table test of large cable-stayed bridge was performed to analyze its modal parameters variations against the earthquake excitations.

5.1. General Introduction of Model Bridge. The model bridge tested in lab was a prototype of actual cable-stayed bridge (scaled down to a ratio of $1: 20$ ). The test bridge span in the laboratory was $6.5+19+6.5 \mathrm{~m}$. The bridge type, its structure layout, floor plan of main girder, and lab model images are shown in Figure 5; as far as the construction materials of lab bridge are concerned, cable tower and its piers were manufactured from normal reinforcement (round steel) with a diameter of $6 \mathrm{~mm}$ and M15 microconcrete, respectively, shear reinforcements were made from 10\# steel wires, both main girder and steel box girder were manufactured from a steel plate with a thickness of $5 \mathrm{~mm}$, and the cable is manufactured from a wire rope with a diameter of $10 \mathrm{~mm}$.

5.2. Location of Acceleration Sensor (Accelerometer). To perform shaking table test for this bridge, four acceleration sensors were installed at the bottom of foundation to monitor the input signals of structure, and eleven accelerometers were installed on the main girder to collect the response signals of structure, as shown in Figure 6.

The sensors used in the cable-stayed bridge are piezoelectric accelerometers, because its working principle is to measure the vibration accelerations by utilizing the characteristics that some special sensitive cores will generate a charge signal proportional to the vibration acceleration. The characteristics of these sensors include the following: wide measuring frequency range, large measuring range, small size, light weight, simple and solidity structure, less external interference, and no need for any external power supply to generate charge signals.

5.3. Earthquake Input. The inputs of earthquake excitation are shown in Table 1 . It can be noticed that the peak acceleration is $0.1 \mathrm{~g}$, the direction of earthquake wave is transverse excitation, and the types of waveforms are Chichi wave and site wave. The acceleration time-history curve of Chichi earthquake wave in $\mathrm{C} 1$ working condition is shown in Figure 7. The sampling frequency of sensors is $256 \mathrm{~Hz}$.

5.4. MIDAS Finite Element Analysis Results. A simulation analysis model of the bridge structure was established using MIDAS 2017. Bridge's piers, towers, and main girders are all simulated using nonlinear beam-column elements, and cables are simulated using truss elements; bearings are all pot-type rubber bearings, and zero-length elements are adopted. The bridge model is shown in Figure 8. The eigenvalue analysis of the bridge was performed, and its first five frequencies are obtained, as shown in Table 2. Figure 9 shows the modal shapes from the first mode to fifth mode.

5.5. Parameter Identification of Each Window. According to the sliding window technology and CDSI algorithm, the modal parameters of the cable-stayed bridge under two types of earthquake excitations are identified. Assume that the initial window time is $1 \mathrm{~s}$, but the appropriate window size is given to be $4 \mathrm{~s}$; additionally, the corresponding sliding window step size is $2 \mathrm{~s}$, which means that window data is normally updated after every $2 \mathrm{~s}$. The signals of each window are identified, and the stability results corresponding to each window in two types of working conditions are obtained. Figure 10 shows the results of the first two stability diagrams in $\mathrm{C} 1$ working condition.

The specific values of the frequency in each window in operating conditions of $\mathrm{C} 1$ and $\mathrm{S} 1$ are identified, and the trend chart of the first five changing frequency values with time is given as shown in Figure 11. Analyzing the frequency changes of each order in the figure, it is shown that the frequency value of the first five orders of bridge structure is stable in both conditions; that is, the bridge structure's natural frequency is stable under the action of two types of earthquake waves. It is also shown that the cable-stayed bridge shows no damage in first two types of conditions.

5.6. Intelligent Identification of Modal Effectiveness. According to the proposed FCM clustering algorithm in Section 3.2, a clustering analysis of each window's parameter results which is under two types of conditions is performed to reach intelligent identification of modal effectiveness in the stability diagram. Figure 12 is the corresponding effective stability diagram of $\mathrm{C} 1$ and $\mathrm{S} 1$ working conditions.

Comparing the MIDAS finite element results with the first five orders of frequency results which are under the conditions of $\mathrm{C} 1$ and $\mathrm{S} 1$, as shown in Table 3, the percent difference between the two results in the table and the errors between the effectiveness modal frequency values obtained 


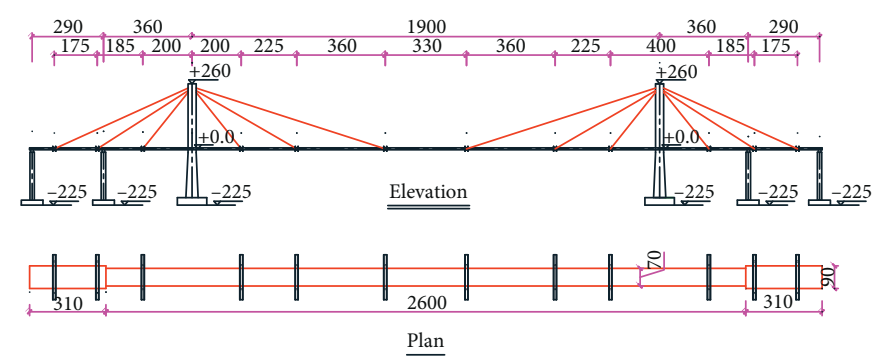

(a)

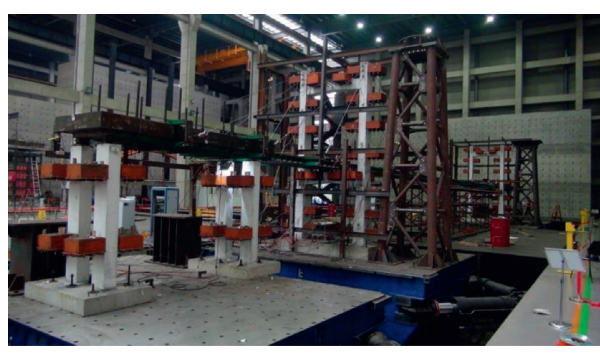

(b)

Figure 5: Bridge model. (a) Elevation and plan (unit: $\mathrm{cm}$ ). (b) Reality image of bridge.

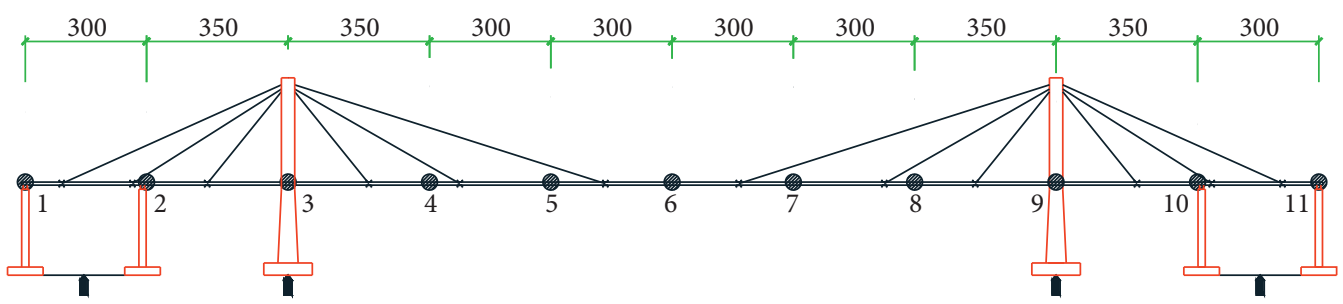

- Acceleration sensors of output

- Acceleration sensors of input

FIgURE 6: Arrangement of acceleration sensors (unit: $\mathrm{cm}$ ).

TABLE 1: Working conditions of test table.

\begin{tabular}{lcccc}
\hline No. & Name & Direction of earthquake wave & Earthquake waveform & Peak acceleration $(\mathrm{g})$ \\
\hline 1 & $\mathrm{C} 1$ & Transverse excitation & Chichi & 0.1 \\
2 & S1 & Transverse excitation & Site wave & 0.1 \\
\hline
\end{tabular}

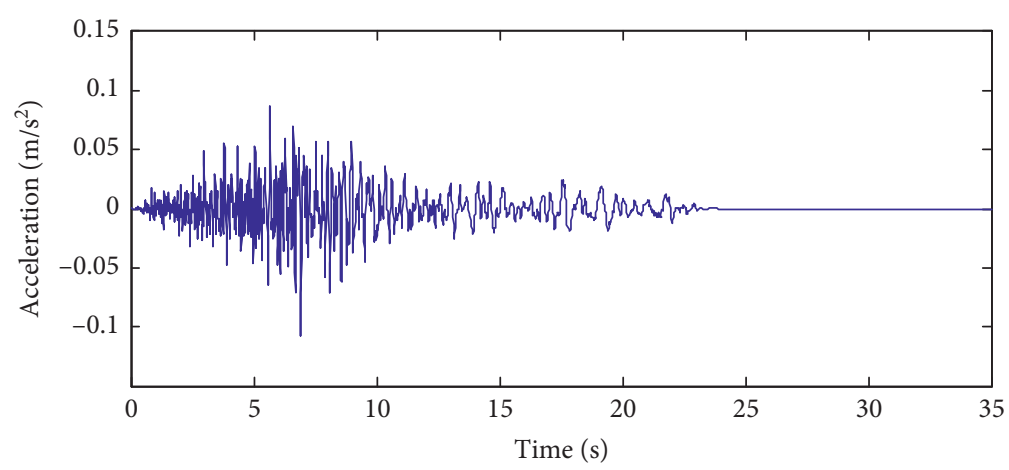

FIgURE 7: Time-history curve of acceleration (C1).

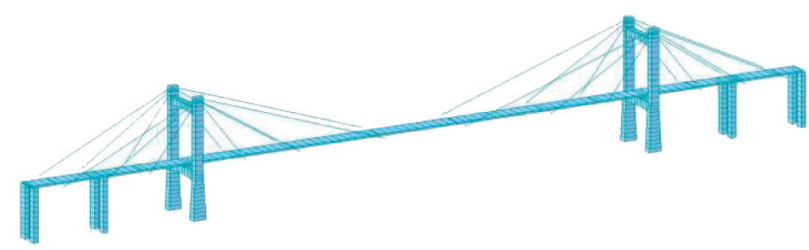

Figure 8: Bridge model (MIDAS). 
Table 2: Natural frequency (unit: $\mathrm{Hz}$ ).

\begin{tabular}{lccccc}
\hline Orders & The first order & The second order & The third order & The fourth order & The fifth order \\
\hline Frequency & 1.14 & 2.51 & 3.77 & 4.63 & 8.83 \\
\hline
\end{tabular}

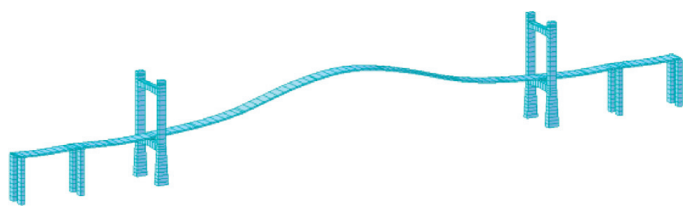

(a)

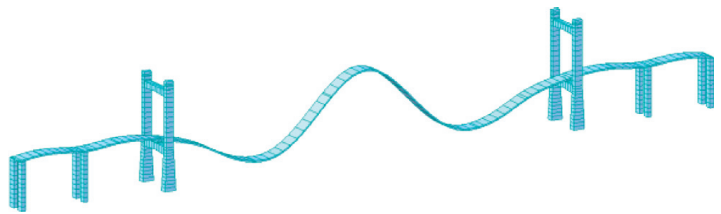

(c)

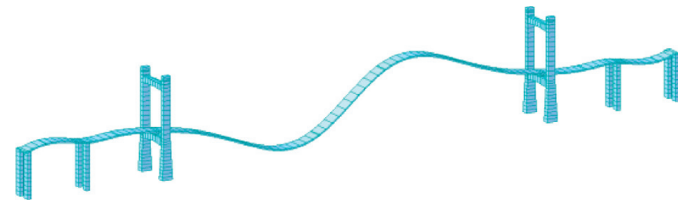

(b)

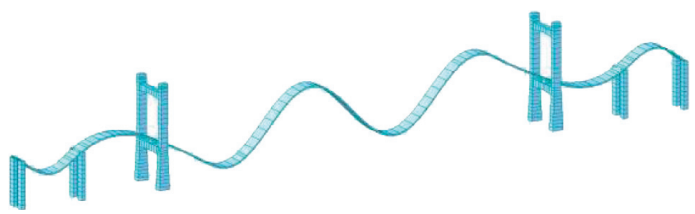

(d)

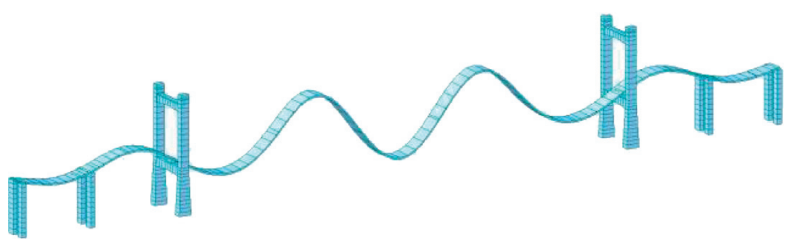

(e)

Figure 9: Modal shape of the first five orders (MIDAS). (a) Vertical bending mode of the first order $\left(f_{1}=1.14 \mathrm{~Hz}\right)$. (b) Vertical bending of the second order $\left(f_{2}=2.51 \mathrm{~Hz}\right)$. (c) Vertical bending of the third order $\left(f_{3}=3.77 \mathrm{~Hz}\right)$. (d) Vertical bending of the fourth order $\left(f_{4}=4.63 \mathrm{~Hz}\right)$. (e) Vertical bending of the fifth order $\left(f_{5}=8.83 \mathrm{~Hz}\right)$.

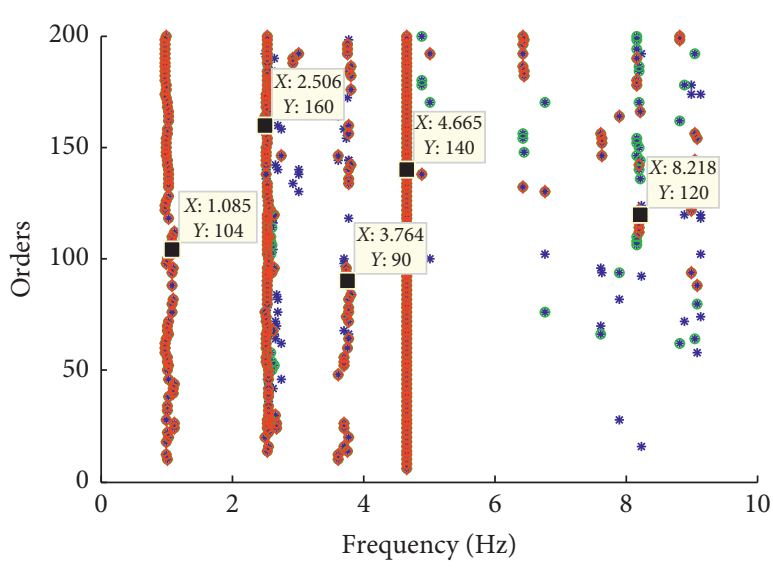

(a)

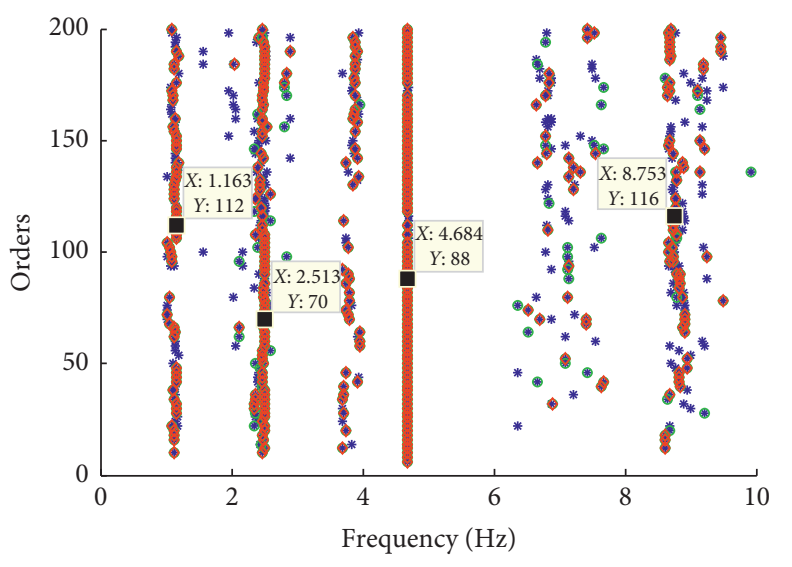

(b)

FIgURE 10: Stability diagram (working condition: C1). (a) Stability diagram (time: $0 \mathrm{~s}-4 \mathrm{~s}$ ). (b) Stability diagram (time: $2 \mathrm{~s}-6 \mathrm{~s}$ ).

by the SC-CDSI algorithm and the finite element results difference are within 7\%; thus, frequency identification results of the proposed SC-CDSI algorithm are hereby considered reliable.

5.7. Modal Shape Results of First Three Orders. Based on these approaches, the SC-CDSI algorithm not only has verified accurately the true modes in the stability diagram but also has identified the modal shapes of structure accurately. Additionally, the first three mode shapes of cable-stayed bridge are obtained, as shown in Figure 13, the X-coordinate in the figure represents the 11 measurement points of sensor, and the Y-coordinate is the mode shape after 0-1 planning; the dotted line is the identification result of the proposed algorithm; the solid line is the mode shape diagram, which is 


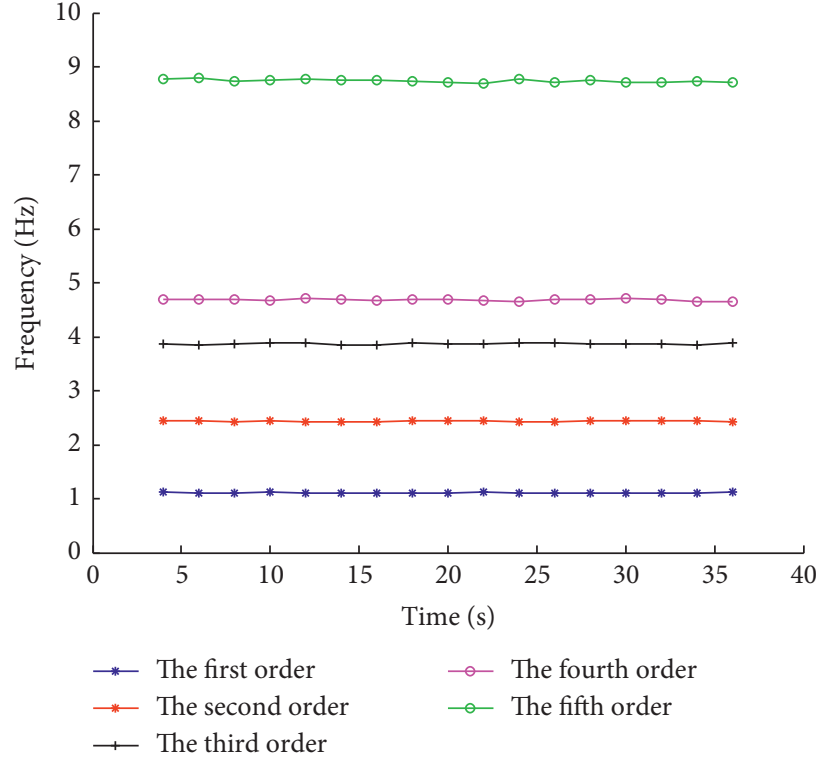

(a)

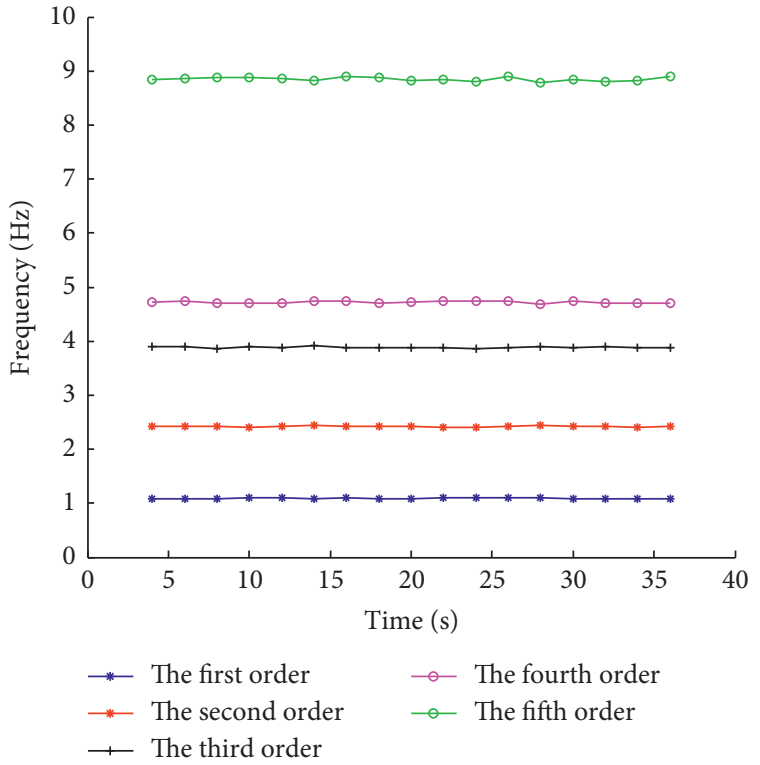

(b)

FIGURE 11: Frequency trend chart. (a) Frequency trend chart (working condition: C1). (b) Frequency trend chart (working condition: S1).

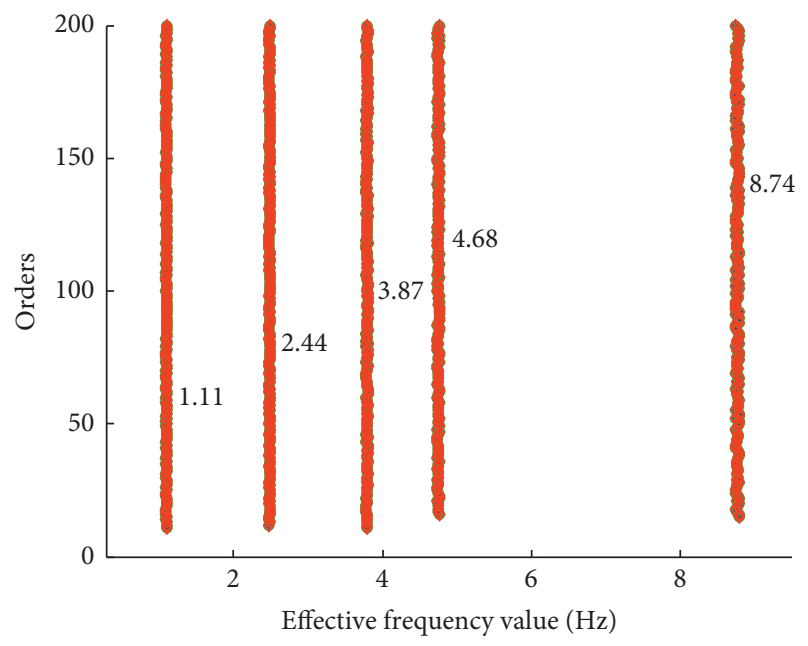

(a)

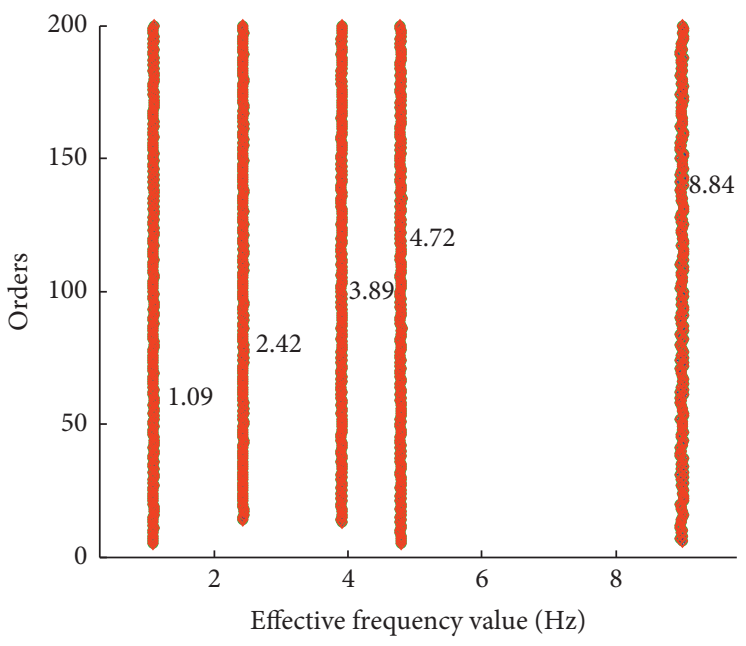

(b)

FIGURE 12: Effective stability diagram. (a) Effective stability diagram (working condition: C1). (b) Effective stability diagram (working condition: S1).

TABLE 3: Comparative analysis results of frequency.

\begin{tabular}{lccccc}
\hline Results & The first order & The second order & The third order & The fourth order & The fifth order \\
\hline MIDAS results $(\mathrm{Hz})$ & 1.04 & 2.56 & 3.77 & 4.54 & 8.43 \\
Working condition: C1 (Hz) & 1.11 & 2.44 & 3.87 & -2.68 & -3.0 \\
Percentage difference 1 (\%) & -6.3 & 4.9 & 3.89 & 4.72 & -3.5 \\
Working condition: S1 (Hz) & 1.09 & 2.42 & -3.1 & -3.8 & -4.6 \\
Percentage difference 2 (\%) & -4.6 & 5.8 & & -4.6 \\
\hline
\end{tabular}

obtained by MIDAS. By comparing these shapes to the first three mode shapes selected from Figure 9, it can be seen that the modal modes and finite element results obtained by the
SC-CDSI algorithm have more than $90 \%$ similarity, which is why the modal shapes obtained by SC-CDSI algorithm are reliable. 


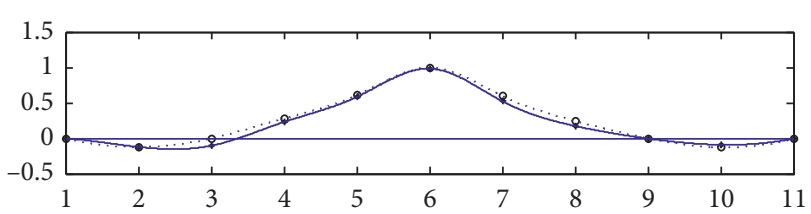

(a)

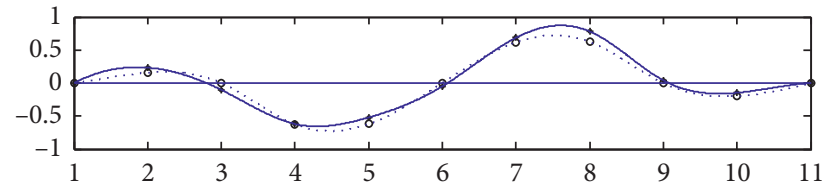

(b)

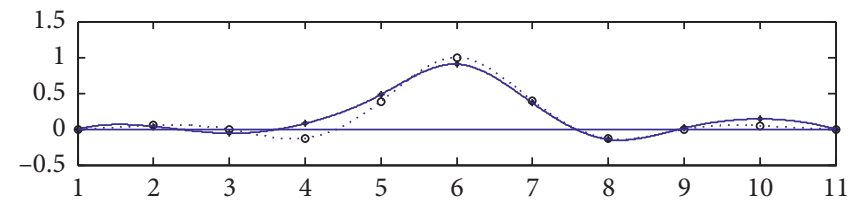

(c)

Figure 13: Modal shape of the first three orders. (a) Vibration diagram of the first order. (b) Vibration diagram of the second order. (c) Vibration diagram of the third order.

\section{Conclusion}

Based on the limitations of deterministic-stochastic subspace algorithm, which is not suitable for nonlinear timevarying structures like bridge, a sliding window technique is proposed in combination with CDSI algorithm to implement the real-time tracking and identification of modal parameters for bridge structures. Additionally, considering that the identification of modal effectiveness in stability diagram needs manual participation, a fuzzy C-means (FCM) clustering algorithm is proposed to identify true and false modes in stability diagram to implement intelligent screening of modal parameter results. Finally, based on the shaking table test of a large cable-stayed bridge in lab, the proposed SC-CDSI algorithm is applied to identify the modal parameters of bridge, and its results are compared with the MIDAS finite element results. The compared results show that the proposed SC-CDSI algorithm not only accurately identifies the natural frequencies values of bridge structure but also accurately identifies the modal shape results and can accurately realize the online intelligent tracking and identification of modal parameters for real-life bridge.

\section{Data Availability}

The data used to support the findings of this paper are freely available. They can be made available on request if and when required.

\section{Conflicts of Interest}

The authors declare that they have no conflicts of interest.

\section{Acknowledgments}

This work was funded by the National Basic Research Program of China (no. 2013CB036302).

\section{References}

[1] M. A. Sanchez, O. Castillo, and J. R. Castro, "Generalized type-2 fuzzy systems for controlling a mobile robot and a performance comparison with interval type-2 and type-1 fuzzy systems," Expert Systems with Applications, vol. 42, no. 14, pp. 5904-5914, 2015.

[2] S. v. Guttula, A. Allam, and R. S. Gumpeny, "Analyzing microarray data of alzheimer's using cluster Analysis to identify the biomarker genes, satya vani guttula, apparao allam," R. Sridhar Gumpeny International Journal of Alzheimer's Disease, vol. 2012, Article ID 649456, 5 pages, 2012.

[3] I. Khan, D. Shan, and Q. Li, "Modal parameter identification of cable stayed bridge based on exploratory data analysis," Archives of Civil Engineering, vol. 61, no. 2, pp. 3-22, 2015.

[4] H. Thai, V. DeBrunner, and L. S. DeBrunner, "Deterministicstochastic subspace identification for bridges," in Proceedings of the Statistical Signal Processing, 2007. SSP'07. IEEE/SP 14th Workshop on IEEE, pp. 749-753, IEEE, Madison, WI, USA, August 2007.

[5] A. Belleri, B. Moaveni, and J. I. Restrepo, "Damage assessment through structural identification of a three-story large-scale precast concrete structure," Earthquake Engineering \& Structural Dynamics, vol. 43, no. 1, pp. 61-76, 2014.

[6] P. G. Bakir, "The combined deterministic stochastic subspace based system identification in buildings," Structural Engineering and Mechanics, vol. 38, no. 3, pp. 315-332, 2011.

[7] M. C. Huang, Y. P. Wang, and M. L. Chang, "Damage detection of structures identified with deterministic-stochastic models using seismic data," The Scientific World Journal, vol. 2014, no. 1, Article ID 879341, 2014.

[8] E. Damore, C. Amaddeo, and A. A. Astaneh, "Health monitoring of an instrumented SRMF building using earthquake data," The Structural Design of Tall and Special Building, vol. 23, no. 13, pp. 980-994, 2014.

[9] B. Moaveni and E. Asgarieh, "Deterministic-stochastic subspace identification method for identification of nonlinear structures as time-varying linear systems," Mechanical Systems and Signal Processing, vol. 31, pp. 40-55, 2012.

[10] P. Lopes dos Santos, J. A. Ramos, and J. L. Martins de Carvalho, "Identification of linear parameter varying systems using an iterative deterministic-stochastic subspace approach," in Proceedings of the Control Conference (ECC), 2007 European, pp. 4867-4873, IEEE, Budapest, Hungary, July 2007.

[11] E. Reynders and G. D. Roeck, "Reference-based combined deterministic-stochastic subspace identification for experimental and operational modal analysis," Mechanical Systems and Signal Processing, vol. 22, no. 3, pp. 617-637, 2008. 
[12] K. P. Yu, S. W. Pang, and J. Zhao, "Advances in method of time-varying linear/nonlinear structural system identification and parameter estimate," Chinese Science Bulletin, vol. 2009, no. 20, pp. 3147-3156, 2009.

[13] T. A. Shen, Y. Q. Tu, H. T. Zhang, and M. Li, "New sliding DTFT algorithm for high-accuracy phase difference measurement based on double rectangle window," Journal of Central South University:Science and Technology, vol. 46, no. 2, pp. 554-560, 2015.

[14] N. A. H. Haldar, F. A. Khan, and A. Ali, "Arrhythmia classification using mahalanobis distance based improved fuzzy c-means clustering for mobile health monitoring systems," Neurocomputing, vol. 220, pp. 221-235, 2017.

[15] D. S. Shan and Q. Li, "Modal parameter identification of bridge structure in time-frequency domain," Bridge Construction, vol. 2015, no. 2, pp. 26-31, 2015.

[16] X. H. Zhou and D. S. Shan, "Sliding window Time-domain method for identifying time-varying modal parameter of multi-input and multi-output bridge structure," Journal of Basic Science and Engineering, vol. 3, pp. 230-242, 2019.

[17] F. Ye, J. Q. Wu, and X. Y. Zhang, "Decomposition and reconstruction of bridge vibration signals based on the harmonic window function," Journal of Vibration and Shock, vol. 16, pp. 234-240, 2018.

[18] C. L. Wu, H. B. Liu, and J. Yu, "Parameter identification of a bridge structure based on a stabilization diagram with fuzzy clustering method," Journal of Vibration and Shock, vol. 32, no. 4, pp. 121-126, 2013.

[19] P. Yan, Research on Noise Reduction and Damage Identification of Bridge Health Monitoring Based on Signal Theory, Southwest Jiaotong University, Chengdu, China, 2011.

[20] B. P. Tang, G. W. Zhang, and Z. Chen, "Automatic identification of stochastic subspace modal parameter based on hierarchical clustering," Journal of Vibration and Shock, vol. 31, no. 10, 2012.

[21] F. Z. Zhang, J. F. Chang, and Q. Q. Zhou, "Context-aware recommendation algorithm based on fuzzy C-means clustering," Journal of Computer Research and Development, vol. 50, no. 10, pp. 2185-2194, 2013.

[22] S. D. Zhou, X. C. Zhou, L. Liu, and W. Yang, "The modal parameters of the automatic verification method based on the fuzzy clustering factors," Journal of Beijing University of Aeronautics and Astronautics, vol. 9, no. 5, 2015. 